\title{
Wh-word conjunction as a test for argumenthood and obligatoriness
}

\author{
Paul B. Melchin \& Ida Toivonen*
}

\begin{abstract}
Some wh-words are easier to conjoin than others. For example, it has been noted that adjunct wh-words are easier to conjoin than argument wh-words. We review previous analyses of wh-word conjunction and evaluate them in light of data collected from a new acceptability judgement study. Our study replicates some previous findings but it also adds some new data to the puzzle. We further revisit the idea that wh-word conjunction can be used as a test for argumenthood, and we conclude that it can, with some caveats. In addition, we conclude that wh-word conjunction can be used as a test for obligatoriness, but only in arguments. The test does not distinguish between obligatory and optional adjuncts.
\end{abstract}

Keywords. Wh-words; argumenthood; syntax; psycholinguistics

1. Introduction. Wh-word conjunction questions (wh-conj) are wh-questions in which there are two (or more) fronted, conjoined wh-words that correspond to different parts of the sentence, as in (1) and (2).

(1) When and where did Mary find the ring?

(2) What and when did John eat?

Not all combinations of wh-words may be involved in wh-conj. Wh-words corresponding to adjuncts (e.g., when and where in (1), when in (2)) may be involved in wh-conj, as can those corresponding to optional objects (specifically, complements of verbs that can undergo the Unspecified Object Alternation (UOA); see Levin 1993, among many others), such as what in (2). However, others may not be involved in wh-conj, including subjects and obligatory objects. This can be seen in (3), where who corresponds to the subject, and in (4), where what corresponds to the object of find (which does not undergo the UOA). ${ }^{1}$

(3) * Who and when ate?

(4) * What and when did John find?

This contrast has led to the use of wh-conj as a diagnostic to test whether a given expression is an argument or an adjunct in a given sentence, as in DeArmond \& Hedberg (1998), Needham $\&$ Toivonen (2011) and Toivonen (2013). However, much of the data is unclear, with speakers and authors on the topic disagreeing on the acceptability of many borderline examples. This leads to difficulty in determining the factors that contribute to the (un)acceptability of wh-conj, which in turn means that it is difficult to determine an appropriate syntactic analysis of the phenomenon. Gračanin-Yuksek (2007) proposes an analysis that essentially predicts that optional

\footnotetext{
* We wish to thank audiences at the LSA Conference and Carleton University Linguistics Reading group for very helpful discussion. We'd also like to thank the anonymous reviewers for their feedback, as well as Raj Singh and Robert Truswell for comments, questions and judgements. This research was funded by SSHRC Insight Grant 4352017-1256. Authors: Paul B. Melchin, University of Ottawa (paul.melchin@uottawa.ca) \& Ida Toivonen, Carleton University (ida.toivonen@carleton.ca).

${ }^{1}$ Note that this paper concerns English only. Wh-conj shows different properties in other languages; for example, the Serbo-Croatian translations of (3) and (4) are both grammatical, while in Dutch, wh-conj is absent entirely (Browne 1973; Gračanin-Yuksek 2007; Citko \& Gračanin-Yuksek 2013); see Citko \& Gračanin-Yuksek (2013) and references therein for extensive discussion of the properties of wh-conj across languages.
} 
elements may be involved in wh-conj, and obligatory ones cannot. However, Lewis et al (2012) provide experimental evidence that even obligatory objects may be wh-conjoined if the whwords are in a certain order, leading Larson $(2013,2015)$ to a different analysis of wh-conj with its own predictions.

In this paper, we set out to determine the empirical scope of wh-conj, and thereby find out if it can be used to diagnose argumenthood or some other property (or properties) of phrases in a sentence. We present results of an acceptability judgement questionnaire; among other results, our findings replicate the findings of Lewis et al (2012). We further show that wh-conj can be used to distinguish arguments from adjuncts, and obligatory arguments from optional arguments, as long as the wh-words are in a certain order. The paper is laid out as follows: In Section 2 we provide a background on wh-conj in English, discussing its past use as an argumenthood diagnostic, the syntactic analysis of Gračanin-Yuksek (2007) and its predictions, the experimental results of Lewis et al (2012) and Larson's (2013, 2015) ensuing analysis, and our own experimental results replicating these findings. With this background established, we next turn to the question of what property or properties wh-conj can test as a syntactic diagnostic. We show that it can be used to diagnose argumenthood, if word order is controlled for; furthermore, among the arguments, it can be used to distinguish obligatory from optional arguments (again, with proper controls for word order). In the following section, we discuss these findings with respect to the relationship between argumenthood and obligatoriness, and speculate on the proper syntactic analysis of wh-conj. In the final section we offer our conclusion.

2. Background on wh-conj. Here we provide a background on wh-conj, discussing its use as an argumenthood diagnostic, a current syntactic analysis of the phenomenon, and a competing analysis resulting from experimental work on the phenomenon, including the results of Lewis et al (2012) and our own replication of their findings.

2.1. WH-CONJ AS A TEST FOR ARGUMENTHOOD. Wh-conj was initially used as a diagnostic for argumenthood by DeArmond \& Hedberg (1998), and was later adopted (with some reservations) by Needham \& Toivonen (2011) and Toivonen (2013, 2016). DeArmond \& Hedberg follow Grimshaw (1990) in assuming that there are three degrees of argumenthood (or more specifically, complementhood, as they exclude subjects from their study): primary complements, which include themes, goals, and other thematic roles generally associated with direct and indirect objects; secondary complements, which are like primary complements in that they are included in the argument structure of the head, but like adjuncts in that they are optional (e.g., instrumental and benefactive phrases); and adjuncts, which are not part of the argument structure of the verb. They claim that the wh-conj test distinguishes between adjuncts on the one hand, for which whconj is possible, and primary and secondary complements on the other, which do not allow whconj. They illustrate this with the following examples (DeArmond \& Hedberg 1998:2-3):

(5) * Who and what did John give to? (goal, theme)

(6) * With what and for whom did John paint the hallway? (instrument, benefactive)

(7) When and how did you find your missing ring? (time, manner)

Crucially, DeArmond \& Hedberg judge example (6), with the two secondary complements, to be ungrammatical. However, not all speakers find this sentence unacceptable, and in fact the participants of our study ranked sentences like (6) relatively highly in comparison with to other whconj examples. It seems likely that to the extent that (6) is degraded compared to (7), it is due to the presence of pied-piped prepositions, which tend to sound unnatural in English (an effect pointed out in other wh-conj examples by Gračanin-Yuksek 2007). 
Thus, wh-conj as a syntactic diagnostic does not clearly distinguish secondary complements from adjuncts. Furthermore, as pointed out by Toivonen (2016), the situation is further complicated by examples such as (2), in which an optional direct object can take part in wh-conj, which is unexpected from the point of view of DeArmond \& Hedberg. Before returning to the question of what wh-conj can diagnose, it is necessary to explore the properties of the phenomenon further. To this end, we turn next to the syntactic analysis of wh-conj proposed by Gračanin-Yuksek (2007) and its predictions.

\subsection{THE SYNTAX OF WH-CONJ. Gračanin-Yuksek (2007) provides an analysis of wh-conj that} accounts for the grammaticality of (1) and (2) and the ungrammaticality of (3) and (4), among other data. She claims that while wh-conj sentences appear to have two wh-words conjoined together, the conjunction is actually between two CPs; the first CP contains the first wh-word, and the second contains the second wh-word and the rest of the sentence. She claims that the material following the wh-word in each $\mathrm{CP}$ is identical, except that it is not pronounced in the first $\mathrm{CP}$. On this analysis, wh-conj is possible only if both CPs are independently well-formed. For examples, in (8) both CPs (what did John eat and what did John devour) are well-formed sentences on their own, while in the ungrammatical (9), only the first $\mathrm{CP}$ is a grammatical sentence, while the second (when did John devour) is not, since devour cannot appear without a direct object.

a. What and when did John eat?

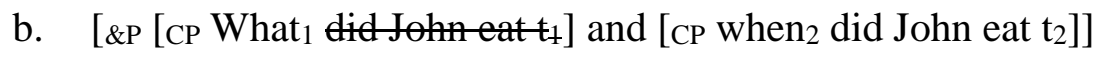

a. * What and when did John devour?

b. [\&P [CP What ${ }_{1}$ did John devour $\left.\mathrm{t}_{1}\right]$ and [CP when 2 did John devour $\left.\left.\mathrm{t}_{2}\right]\right]$

Gračanin-Yuksek's analysis of wh-conj involves multidominance structures (or, in her terms, "sharing" structures), in which the unpronounced material in the first CP is not deleted, but is instead "shared" between the two CPs, being present in both but pronounced only in the second. In this analysis, each of the terminal nodes (in (8b), the lexical items did, John, eat, and the trace) is dominated by two mother nodes, one in each CP. She outlines constraints on this kind of structure (collectively dubbed the Constraint on Sharing, $\mathrm{COSH}$ ) that ensures that the two CPs are identical (at least in their terminal nodes) and well-formed, as well as a linearization algorithm to ensure that the shared material is pronounced in the second CP. We will not discuss these details here, though we will discuss the empirical predictions of the analysis. See GračaninYuksek (2007) and Citko \& Gračanin-Yuksek (2013) for more on the analysis itself.

Gračanin-Yuksek's sharing analysis of wh-conj makes the following predictions:

- optional items (optional objects, adjuncts) can be wh-conjoined;

- subjects cannot be wh-conjoined;

- preposition stranding is impossible in wh-conj ${ }^{2}$

- obligatory arguments cannot be wh-conjoined;

- the order of the conjuncts does not affect grammaticality.

\footnotetext{
2 A possible exception to this prediction (which is not mentioned by Gračanin-Yuksek) is that it should be possible if both wh-words strand the same preposition. For example, in the sentence, ?? What time and what theatre did Alex see the movie at?, the two wh-phrases (what time and what theatre) both strand the preposition at. It is unclear to us whether or not this prediction holds; the participants in our survey gave mixed results to this kind of sentence, though this may be due to the fact that it is quite difficult to construct sentences of this form that sound natural in the first place. It is also difficult to find examples where both adjuncts require (not just allow) a preposition. For example, the at is optional in At what time did Alex see the movie?
} 
The first and fourth predictions are discussed above. The impossibility of subjects in wh-conj is predicted both because subjects are generally obligatory in English, and because the structure of subject wh-questions in English is different from other wh-questions in that it does not involve auxiliary movement, and so the two CPs could never be identical. Preposition stranding is impossible since it the stranded preposition would only be grammatical in one conjunct (though see Footnote 2 for an exception to the prediction); for example, in (10), for both conjuncts to be identical, both must have a stranded with, which is ungrammatical in the first conjunct. In (11), with no stranded preposition, both conjuncts are grammatical.

(10) a. * When and who(m) did John eat with?

b. [\&P [CP When 1 did John eat with $\left.\mathrm{t}_{1}\right]$ and [CP who(m) $)_{2}$ did John eat with $\left.\left.\mathrm{t}_{2}\right]\right]$

(11) a. When and with who(m) did John eat?

b. [\&P [CP When 1 did John eat $\left.\mathrm{t}_{1}\right]$ and [CP with who(m) $)_{2}$ did John eat $\left.\left.\mathrm{t}_{2}\right]\right]$

Of these predictions, the first three appear to hold (with the caveat mentioned in Footnote 2). However, the last two, regarding the possibility of obligatory arguments in wh-conj and the order of conjuncts, have been shown not to hold in experimental studies. It is to these studies that we now turn.

2.3. OTHER ANALYSES. In an experimental study, Lewis, Larson \& Kush (2012) demonstrate that in wh-conj sentences with verbs that take an obligatory object, such as devour or find, the order of the two wh-words has an effect on grammaticality. While these sentences are ungrammatical when the wh-word corresponding to the direct object is in the first conjunct, as shown in (4) above and repeated in (12a), Lewis et al show that they are acceptable when the object wh-word is in the second conjunct $(12 b)$ :
a. * What and when did John find?
b. When and what did John find?

This is demonstrated experimentally using speeded acceptability judgement and self-paced reading tasks. They tested both obligatory object verbs, such as find, and optional object verbs, such as eat, and for each they tested both orders of the wh-words. In both tasks, for the optional-object verbs they found no significant effect of order, while the obligatory-object verbs did show a significant effect of order. For these verbs, the judgement scores and reading times for the objectsecond order (as in (12b)) were similar to those for the optional-object verbs, while the results for the object-first order (as in (12a)) were significantly lower. In other words, for optionalobject verbs, the order of the wh-words does not affect acceptability, as shown in (13). On the other hand, obligatory objects are only disallowed in wh-conj when they are in the first conjunct, while in the second conjunct they pattern like optional objects.

\section{(13) a. What and when did John eat? \\ b. When and what did John eat?}

An analysis for these facts is proposed in Lewis et al (2012), and a version of it developed in Larson $(2013,2015)$, according to which the verb has two different kinds of relationships with the two wh-words, contrary to the proposal of Gračanin-Yuksek (2007). In this account, wh-conj is still the conjunction of two CPs; however, only the second is a full CP, while the first contains only the (null) $\mathrm{C}$ head and the wh-word in its specifier and no deleted material, as shown in (14) for the sentence in (13a):

$$
\left[\& \mathrm{P}[\mathrm{CP} \text { what } \mathrm{C}] \text { and }\left[\mathrm{CP} \text { when }_{1} \mathrm{C} \text { did John eat } \mathrm{t}_{1}\right]\right]
$$


The wh-word in the specifier of the second CP is in that position as a result of normal whmovement from lower in the phrase, while the second is base-generated in spec-CP and is not related to any trace (or copy) elsewhere in the $\mathrm{CP}$. It gains its interpretation as an argument or adjunct to the $\mathrm{V}(\mathrm{P})$ in the second conjunct not from the usual syntactic and semantic mechanisms associated with wh-movement, but instead through some non-syntactic, possibly nongrammatical, mechanism.

As for the identity of the means by which the first wh-word gains its interpretation, Larson (2013) argues that it is not any syntactic, semantic, or pragmatic mechanism. Any relevant syntactic relationship between what and eat in (14) is blocked by the lack of c-command between the two. Similarly, semantic binding relationships are blocked because what does not scope over eat. On the other hand, a pragmatic relationship in the form of a discourse dependency between the two items overproduces, as the kind of dependency that would allow (14) would also rule in sentences such as (15a), with a meaning along the lines of (15b) (Larson 2013:62): ${ }^{3}$

a. * What and when did Becky eat the cake?

b. What is it such that Becky ate it and the cake?

Larson concludes that there is some (as-yet unidentified) non-grammatical, psycholinguistic effect is at play in the interpretations of these structures; that is, it is a matter of linguistic performance, rather than linguistic competence.

2.4. OUR RESULTS: ORDER EFFECTS. The difference between Gračanin-Yuksek's (2007) account of wh-conj and that of Larson (2013) relies crucially on the difference in grammaticality between sentences like (12a) and those like (12b), repeated in (16).

a. * What and when did John find?

b. When and what did John find?

The acceptability of (16b), in which a wh-word corresponding to an obligatory object is acceptable in wh-conj, is unexpected in Gračanin-Yuksek's account, as is the mere fact that the order of conjuncts should affect the grammaticality of these sentences. It is difficult to see how the sharing-based account of Gračanin-Yuksek could be modified in order to account for these facts. In fact, the effect of the order of conjuncts poses a problem to any purely syntactic or semantic account of wh-conj. However, the contrast in (16), as with many judgements related to wh-conj, is not a clear distinction between a totally acceptable and totally unacceptable option for many speakers. Rather, the judgements are more gradient and vary somewhat between speakers. In other words, the claims of Lewis et al (2012) and of Larson $(2013,2015)$ are based on the crucial observation that (16b) is acceptable to the same degree as parallel sentences with optional-object verbs (e.g., (13)), contrary to the predictions of Gračanin-Yuksek.

In order to verify these claims and investigate data relevant to certain other aspects of whconj, we performed an experiment in which we gathered acceptability judgement data on whconj with a variety of verbs and wh-words, with both possible orders of conjuncts. Here we discuss the experimental results that are relevant to the current discussion; other results will be discussed in Section 3 as well. The experiment consisted of two acceptability judgement questionnaires, each with the same sentences except with the wh-words reversed such that each participant saw sentences with the wh-words in both orders, but saw only one order for each

\footnotetext{
${ }^{3}$ For reasons of space we leave out the details of each of these arguments; see Larson (2013:48-63) for extensive discussion.
} 
verb. The surveys were distributed to students at Carleton University, with the 52 participants responding to the first survey and 59 to the second. A 7-point Likert scale was used. The experiment was a pilot study, investigating many different variables with only a few different verbs in each category. However, it produced some significant results, including a robust replication of Lewis et al's (2012) findings.

Our experiment included the optional-object verbs eat, sing, and bake, and the obligatoryobject verbs deny, find, devour, and buy, each with both orders of wh-words. We found that, on the whole, the obligatory-object verbs $(\mathrm{M}=3.7, \mathrm{SD}=1.9)$ received lower ratings than optionalobject verbs $(\mathrm{M}=4.2, \mathrm{SD}=1.8)$; we conducted a Welch two-sample t-test and found this difference to be significant $(t(884)=4.41, \mathrm{p}<.001)$. However, as illustrated in Figure 1, there is an interaction between optionality and word order (an ANOVA showed the interaction to be significant, $\mathrm{p}<0.001)$. In particular, we found that in the object-second order, there is no significant difference between obligatory-object and optional-object verbs ( $M=4.3$ and $M=4.32$, respectively), while in the object-first order, obligatory-object sentences receive significantly lower scores $(\mathrm{M}=3.15$, compared to $\mathrm{M}=4.16$ for optional-object verbs).

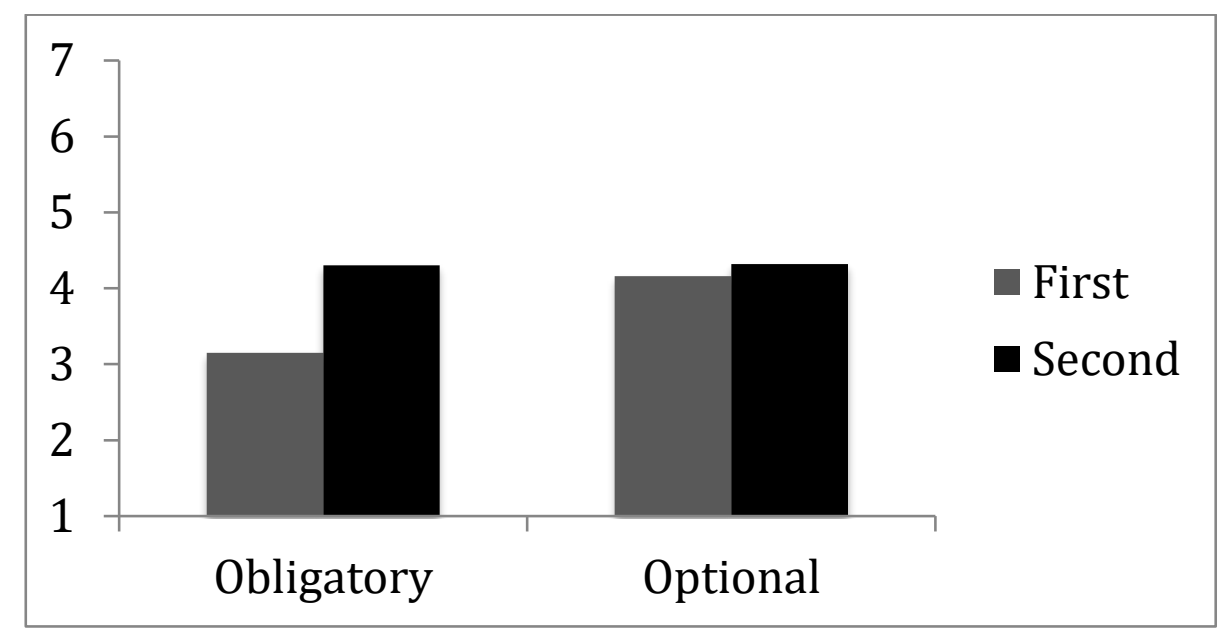

Figure 1. Interaction between word order and optionality in arguments

In other words, our results demonstrated the paradigm in (17)-(18), replicating the findings of Lewis et al (2012):

(17) a. * What and when did John find?

b. When and what did John find?

(18) a. What and when did John eat?

b. When and what did John eat?

Note, however, that the scores (out of 7) are not particularly high for any of these argument types, regardless of the word order. As we will see below, adjunct wh-words were overall ranked higher.

In Section 4 we discuss some consequences of these and other findings for the potential analyses of wh-conj. First, however, we return to the main research question of this investigation: Is wh-conj a useful diagnostic? If so, for what?

3. Wh-conj as a diagnostic. Recall the claim of DeArmond \& Hedberg (1998), that wh-conj can be used as a diagnostic to distinguish between arguments (or in their terms, primary and second- 
ary complements), for which wh-conj is impossible, and adjuncts, for which it is possible. The discussion above and the references cited make clear that the situation is not so straightforward. Judgements for wh-conj differ according to whether the verb takes an obligatory or optional object, as well as the order of the wh-words. In addition, acceptability judgements for wh-conj sentences tend not to be absolute, but are rather gradient, requiring experimental testing to tease apart some subtle distinctions. Thus, as a straightforward syntactic test, where a grammatical sentence diagnoses one property and an ungrammatical sentence diagnoses another, wh-conj is of limited use. However, when experimental results are taken into account, wh-conj can be used to test for both argumenthood and obligatoriness. Before discussing the relevant experimental results, a brief discussion of the relationship between argumenthood and obligatoriness is in order.

Generally speaking, argumenthood and obligatoriness are considered to be intertwined. While certain kinds of arguments may be optional, as we have seen above, adjuncts are generally considered to be universally optional (Tallerman 2005:98); if something looks like an adjunct but is obligatory, then it is generally considered to be an argument instead, as with the locative PP argument of put:

(19) John put the book*(on the table).

However, there are certain instances where some kind of adjunct is necessary in order for the sentence to be acceptable (Grimshaw \& Vikner 1993; Goldberg \& Ackerman 2001; Sailor \& Schutze 2013). This is particularly common with certain kinds of passive and middle sentences, as in (20) (examples adapted from Goldberg \& Ackerman 2001) ${ }^{4}$ :

(20) a. This house was built \#(last year/from brick/by Mary).

b. This book reads \#(easily/slowly).

These examples illustrate that, like arguments, adjuncts too can be either obligatory or optional. In other words, obligatoriness and argumenthood are orthogonal properties, crosscutting one another. Now recall the prediction of Gračanin-Yuksek's (2007) analysis, that wh-conj is possible for optional elements but impossible for obligatory ones; while this holds for arguments (at least in one order of conjuncts), this does not hold for obligatory adjuncts, for which wh-conj is always possible:

(21) a. This house was built \#(last year/from brick/by Mary).

b. When and how was this house built?

(22) a. The best tomatoes are grown \#(in Italy/with lots of water/with great care).

b. How and where are the best tomatoes grown?

This may be expected on Gračanin-Yuksek's sharing analysis, given the fact that sentences that require an adjunct are generally not picky about what kind of adjunct is added. If the structure of

\footnotetext{
${ }^{4}$ Analyses of obligatory adjuncts vary; Grimshaw \& Vikner (1993) propose an event structure-based account, while Goldberg \& Ackerman (2001) claim it is due to pragmatic, Gricean inference. Sailor \& Schutze (2013) reject both analyses without offering an alternative. While we do not attempt to resolve this issue here, it is worth mentioning that sentences like those in (20) can often be made felicitous in the absence of an adjunct by changing the tense/aspect/mood of the sentence (This house will have been built; This house should be built) or by adding negation or emphasis (This house wasn 't built; This house WAS built), as noted by Goldberg \& Ackerman (2001), suggesting it is not an adjunct per se that licenses the sentences, but instead anything that makes the sentence more informative than a simple statement, supporting their pragmatic analysis. For the purposes of this paper we abstract away from these issues and treat the adjuncts in (20) simply as obligatory.
} 
(21b) is as in (23), as in the sharing analysis, then both CPs are expected to be grammatical, as in each there is some item modifying (and thereby licensing) the sentence.

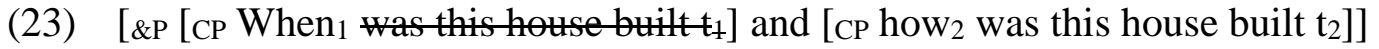

Larson's $(2013,2015)$ analysis also predicts that they will be grammatical. Nevertheless, whconj drives home the following two points: (a) obligatoriness and argumenthood are orthogonal; and (b) adjunct obligatoriness is different from argument obligatoriness. Verbs require very specific types of obligatory arguments (e.g., themes), but verbs that require adjuncts do not require adjuncts of a particular type.

Returning to our experimental results, we find that abstracting away from the effects of the order of wh-words, wh-conj can be used to distinguish between arguments and adjuncts, and among the arguments, it can distinguish between obligatory and optional arguments (though it does not diagnose obligatoriness among the adjuncts). Using t-tests (Welch two-sample), we found a significant difference between the acceptability scores for arguments and adjuncts as a whole $(\mathrm{t}(1458)=11.7, \mathrm{p}<.001)$, as well as a difference between obligatory arguments $(\mathrm{M}=3.74$, $\mathrm{SD}=1.91)$ and optional arguments $(\mathrm{M}=4.24, \mathrm{SD}=1.72 ; \mathrm{t}(884)=4.41, \mathrm{p}<.001)$. However, there was no significant difference between obligatory adjuncts $(\mathrm{M}=4.98, \mathrm{SD}=1.77)$ and optional adjuncts $(\mathrm{M}=5.15, \mathrm{SD}=1.66 ; \mathrm{t}(651)=1.27, \mathrm{p}=0.2)$. These results are shown in Figure 2.

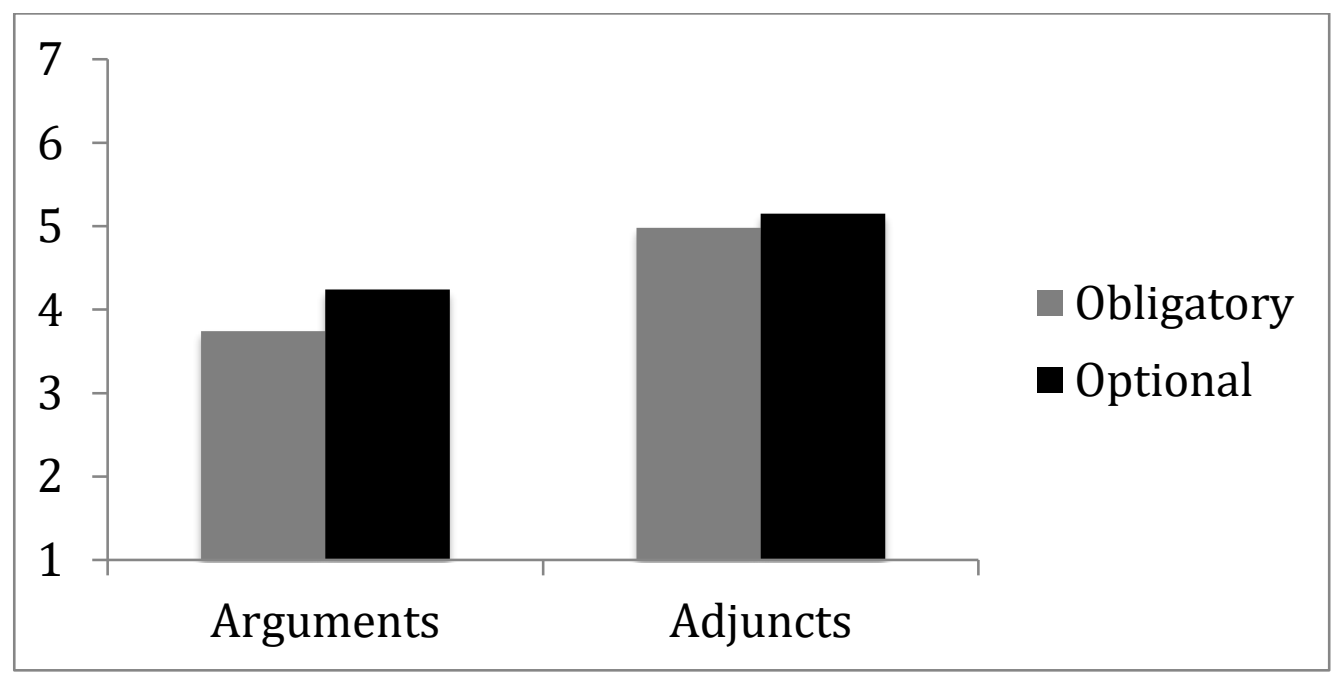

Figure 2. Obligatory and optional arguments and adjuncts (ordering conflated)

Thus, if the appropriate care is taken with the testing, wh-conj can be used as a diagnostic to test for argumenthood, and among the arguments, to test for obligatoriness. However, the contrast between obligatory and optional arguments is visible only when the argument wh-word comes first, not when it comes second (in contrast, the argument-adjunct distinction is visible in either order). Furthermore, due to the subtlety and gradience of the judgements involved, they are often only apparent in an experimental context, when many speakers' judgements are taken into consideration.

Having established the properties that wh-conj diagnoses, the question might be asked why it is necessary to have a syntactic test to diagnose obligatoriness. It is generally assumed that it is easy to tell whether an element in a sentence is optional, as it can be omitted from the sentence without making the sentence ungrammatical. However, it has been noted (by, e.g., Goldberg 
2005; Mittwoch 2005; Glass 2014) that there are contexts in which so-called obligatory objects can be omitted, as in the following examples (Goldberg 2005, via Glass 2014:123):

(24) a. Tigers only kill at night.

b. The singer always aimed to please/impress.

c. He burglarized, but she murdered!

This is only possible in certain, well-defined contexts, such as generic sentences (24a), infinitives (24b), and emphasis or contrastive focus (24c). However, they serve to illustrate that the line between obligatory and optional arguments can be blurred. Wh-conj can be used to abstract away from the context and unambiguously distinguish the two.

4. Discussion and consequences. This study has a number of theoretical consequences. One such consequence, hinted at in the above discussion, is that our results reinforce the notion that argumenthood and obligatoriness are orthogonal properties. That is, contrary to the simplistic view often espoused by syntax textbooks, it is not the case that all arguments are obligatory, and all adjuncts are optional. There are optional objects, and there are constructions where some adjunct is necessary. While this is not news, it is useful to have a syntactic test that can distinguish obligatoriness and argumenthood without being confounded by certain niche contexts, such as those illustrated in (24).

In addition, this research adds to the discussion of English wh-conj, contributing to the empirical scope of the phenomenon and therefore to the possible analyses. Recall from Section 2 that Gračanin-Yuksek's (2007) sharing analysis of the phenomenon leads to certain predictions, two of which were found not to hold by Lewis et al (2012) - namely, wh-conj is possible with obligatory objects under certain conditions, with the order of conjuncts unexpectedly affecting grammaticality. Our experimental results replicate Lewis et al.'s findings, further casting doubt on the sharing analysis. Furthermore, in both Lewis et al.'s data and our own, there is gradience in the data that is unexpected in any syntactic account: if wh-conj is possible in one kind of syntactic configuration but not in others, then it is expected that a sentence with this configuration will be fully grammatical/acceptable, and others will be entirely ungrammatical/unacceptable. The gradience involved suggests that extra-syntactic factors are involved, as proposed by Lewis et al (2012) and Larson $(2013,2015)$.

However, some of the experimental results are difficult to explain under Larson's analysis as well. For example, even in the second conjunct there is a contrast between adjuncts and obligatory arguments ${ }^{5}$; thus, it seems hasty to claim that the relationship between the first conjunct and the verb is entirely non-syntactic. Furthermore, we found ordering effects that are unexpected in any of the analyses reviewed so far. Specifically, there are some instances where the order of two adjunct wh-words has a notable effect on acceptability judgements. Since we are not sure how to divide these examples into a coherent subclass, we have not tested for significance. However, a sizeable difference in mean judgement score is visible in the following examples:

(25) a. How and when was this house built?

$(\mathrm{M}=5.54)$

b. ?*When and how was this house built?

$(\mathrm{M}=4.54)$

(26) a. Where and how was this building designed?

$(\mathrm{M}=5.14)$

b. ?*How and where was this building designed?

$(\mathrm{M}=4.19)$

a. When and why did Pat leave the party?

$(\mathrm{M}=5.72)$

${ }^{5}$ Obligatory arguments in second position: $\mathrm{M}=4.3$, all adjuncts (obligatory and optional): $\mathrm{M}=5.08(\mathrm{t}(405)=5.5$, $\mathrm{p}<0.001)$. 
b. ?*Why and when did Pat leave the party?

$(\mathrm{M}=4.83)$

It is not clear to us how these differences could be explained on any analysis of wh-conj. ${ }^{6}$ One possibility is that speakers are used to hearing wh-words coordinated in certain orders more commonly than in others, and that these frequency effects are contributing to how acceptable they find wh-conj sentences; a corpus study would be necessary to test this hypothesis. While a more detailed analysis is needed to determine when and how the order of conjuncts affects the acceptability of wh-conj, the data we have suggests that a multitude of factors contribute to the availability of wh-conj, which may include syntactic, semantic, and pragmatic factors, frequency of collocations, plausibility of the situation depicted by the sentence, and so on.

5. Conclusion. We conclude that wh-conj can be used to distinguish between arguments and adjuncts, although the contrast is only obvious with obligatory arguments. Adjuncts can participate in wh-conj whether they are obligatory or optional. It thus seems that wh-conj can be used to diagnose argumenthood and obligatoriness in combination. Our results support the conclusion of Larson $(2013,2015)$ that the multidominance-based syntactic analysis (Gračanin-Yuksek 2007; Citko \& Gračanin-Yuksek 2013) goes a long way but ultimately does not correctly account for the distribution of wh-conj. However, we found additional effects of the ordering of the whconjuncts that Larson's $(2013,2015)$ analysis does not account for. It seems that a number of factors, including syntactic and discourse factors, are relevant for a full understanding of the distribution of conjoined wh-words.

\section{References}

Browne, E. Wayles. 1972. Conjoined question words and a limitation on English word structures. Linguistic Inquiry 3. 223-226. http://www.jstor.org/stable/4177703

Citko, Barbara \& Martina Gračanin-Yuksek. 2013. Towards a typology of coordinated whquestions. Journal of Linguistics. 49. 1-32. https://doi.org/10.1017/S0022226712000175

DeArmond, Richard C. \& Nancy Hedberg. 1998. On complements and adjuncts. Proceedings of CLA 1998.

Glass, Lelia. 2014. What does it mean for an implicit object to be recoverable? U. Penn Working Papers in Linguistics 20(1). 121-130. https://repository.upenn.edu/pwpl/vol20/iss1/14

Goldberg, Adele. 2005. Constructions, lexical semantics, and the Correspondence Principle: Accounting for generalizations and subregularities in the realization of arguments. In Nomi Erteschik-Shir \& Tova Rapoport (eds.), The Syntax of Aspect, 215-236. Oxford: Oxford University Press. https://doi.org/10.1093/acprof:oso/9780199280445.003.0010

Goldberg, Adele \& Farrell Ackerman. 2001. The pragmatics of obligatory adjuncts. Language 77. 798-814. https://doi.org/10.1353/lan.2001.0219

Gračanin-Yuksek, Martina. 2007. On Sharing. Cambridge, MA: MIT dissertation.

Grimshaw, Jane. 1990. Argument Structure. Cambridge, MA: MIT Press.

\footnotetext{
${ }^{6}$ Janet Randall (p.c.) has suggested that the judgements in (25)-(27) may be due to superiority effects, where the less acceptable examples involve crossing dependencies (i.e., the first wh-word corresponds to the first adjunct and the second wh-word to the second adjunct), while the better examples have nested dependencies. If so, this could be a factor that affects the grammaticality of wh-conj. However, Citko \& Gračanin-Yuksek (2013) show that English whconj does not show superiority effects in general. Furthermore, it is not clear that there is any order of non-wh adjuncts that is strongly preferred in sentences like (25)-(27), and so it is difficult to confirm whether or not any of the examples involve crossing dependencies.
} 
Grimshaw, Jane \& Sten Vikner. 1993. Obligatory adjuncts and the structure of events. In Eric Reuland and Werner Abraham (eds.), Knowledge and Language, Vol. II, 143-155. Dordrecht: Kluwer. https://doi.org/10.1007/978-94-011-1842-2_7

Larson, Brooke. 2013. The Syntax of Non-Syntactic Dependencies. College Park, MD: University of Maryland dissertation.

Larson, Brooke. 2015. Minimal search as a restriction on Merge. Lingua 156. 57-69. https://doi.org/10.1016/j.lingua.2014.12.009

Levin, Beth. 1993. English Verb Classes and Alternations. Chicago: University of Chicago Press.

Lewis, Shevaun, Brooke Larson \& Dave Kush. 2012. What and when can you fill a gap with something? Presented at CUNY sentence processing conference.

Mittwoch, Anita 2005. Unspecified Arguments in Episodic and Habitual Sentences. In Nomi Erteschik-Shir \& Tova Rapoport (eds.), The Syntax of Aspect, 237-254. Oxford: Oxford University Press. https://doi.org/10.1093/acprof:oso/9780199280445.003.0011

Needham, Stephanie \& Ida Toivonen. 2011. Derived arguments. In Miriam Butt \& Tracy Holloway King (eds.), Proceedings of the LFG11 Conference, 401-421. Stanford: CSLI Publications.

Sailor, Craig \& Carson T. Schütze. 2013. What is an adjunct? Ms. http://ling.auf.net/lingbuzz/002180/

Tallerman, Maggie. 2005. Understanding Syntax, 2nd ed. London: Hodder Arnold.

Toivonen, Ida. 2013. English benefactive NPs. In Miriam Butt \& Tracy Holloway King (eds.), Proceedings of the LFG13 Conference, 503-523. Stanford: CSLI Publications.

Toivonen, Ida. 2016. Revisiting the argument-adjunct distinction. Handout from talk at Stanford University.

Whitman, Neal. 2004. Semantics and pragmatics of English verbal dependent coordination. Language 80. 403-434. http://www.jstor.org/stable/4489719 